\title{
Improvement on LEACH Protocol of Wireless Sensor Network
}

\author{
Xiaowen Ma \\ Institute of Telecom Services Support Systems \\ Research, Chongqing University of Posts and \\ Telecommunications \\ Chongqing, China \\ E-mail: maxiaowen0601@126.com
}

\author{
Xiang $\mathrm{Yu}$ \\ Institute of Telecom Services Support Systems \\ Research, Chongqing University of Posts and \\ Telecommunications \\ Chongqing, China \\ E-mail: xiangyu@cqupt.edu.cn
}

\begin{abstract}
Wireless sensor networks comprise of minor battery driven devices with restricted energy resources.Once installed,the minor sensor nodes are usually unapproachable to the operator, and thus auxiliary of the energy source is not practicable.Hence,energy proficiency is a vital design issue that needs to be boosted in order to increase the lifetime of the network. LEACH is a popular hierarchical routing protocol which efficiently maintains the energy storage of nodes in Wireless Sensor Network(WSN).The nodes using LEACH are divided into clusters. The advantage of LEACH is that each node has the equal probability to be a cluster head,which makes the energy dissipation of each node be relatively balanced. This paper studies LEACH protocol, and focuses on how to decide the next hop nodes more reasonable when the data are transmitted at the steady state. Simulation has been done in NS2 and the results show that the algorithm after improved is more energy-efficient than LEACH protocol.
\end{abstract}

Keywords- LEACH protocol; energy; lifetime; Wireless sensor networks

\section{INTRODUCTION}

Sensor networking is a thought-provoking research area that attracts contributions from distributed algorithms, signal processing, networking and protocols, databases and information management, and architecture. Wireless Sensor Network(WSN)has been widely deployed in the applications of military, science research, industry and environment protection[1].

The wireless sensor networks can be installed in an extensive geographical space to observe physical phenomenon with adequate precision and dependability. The sensors can observe numerous objects such as: temperature, pressure, metallic objects, and motion. Sensor nodes may impulsively form network, assemble the network themselves, animatedly familiarize to device let-down and deprivation, manage movement of sensor nodes, and respond to variations in job and network necessities. Every individual sensor comprises both processing and communication elements and is intended to observe the surroundings for events identified by the operator of the network.

That's why Sensor networks have an extensive variety of uses and systems with massively unpredictable requirements and features. The sensor networks can be used in Military situation, Disaster management, Habitat observing, Medical and health care, Industrial fields, Home networks, Spotting chemical, Biological, and Explosive material etc. Placement of a sensor network in these applications can be fixed manually. Interfacing these sensors can assist rescue operations by tracing survivors, recognizing dangerous areas, and making the rescue team more conscious of the overall condition in the disaster area[2].

The basic idea of clustering routing[3] is to use the information aggregation mechanism in the cluster head to reduce the amount of data transmission, thereby, reduce the energy dissipation in communication and in turn achieve the purpose of saving energy of the sensor nodes. In the clustering routing algorithms for wireless networks, LEACH(low-energy adaptive clustering hierarchy [1][4])is well-know because it is simple and efficient. LEACH divides the whole network into several clusters, and the run time of network is broken into many rounds. In each round, the nodes in a cluster contend to be cluster head according to a predefined criterion. In LEACH protocol, all the sensor nodes have the same probability to be a cluster head, which makes the nodes in the network consume energy in a relatively balanced way so as to prolong network lifetime.

\section{LITERATURE SURVEY AND RELATED WORKS}

\section{A. Classification of Routing Protocols}

On the basis of Network Structure routing protocols in WSNs can be divided into location-based routing hierarchical-based routing, flat-based routing. In flat-based routing, all nodes are assigned identical tasks. In hierarchical-based routing, nodes will perform dissimilar tasks in the network. In location-based routing, sensor nodes' positions are utilized to route data in the network. Moreover, on the basis of Operation protocols can also be classified into negotiation-based, multipath-based, querybased, Qos-based, or coherent-based routing techniques. The classification of Routing protocols in WSNs is shown in Fig.1. 


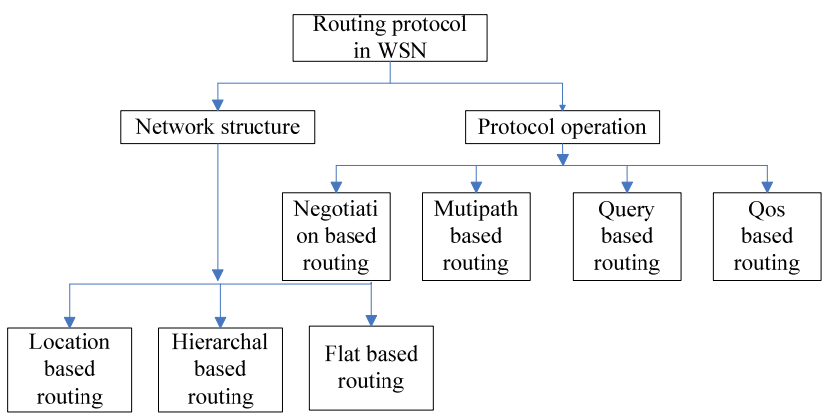

Figure 1. Classification of routing protocols in WSN

\section{B. LEACH protocol}

LEACH is an adaptive clustering routing protocol proposed by Wendi B. Heinemann, et al. In LEACH the purpose is estranged into rounds, during each round a different set of nodes are cluster-heads $(\mathrm{CH})$. The implementation process of LEACH includes many rounds. Each round consists of the set-up phase and the steady data transmission phase. In the set-up phase, the cluster head nodes are randomly selected from all the sensor nodes and several clusters are constructed dynamically. In the steady data transmission phase, member nodes in every cluster send data to their own cluster head, the cluster head compresses the data that received from member nodes and sends the compressed data to the sink node. LEACH protocol periodically elects the cluster head nodes and reestablishes the clusters according to a round time, which ensures energy dissipation of each node in the network is relatively evenly.

The cluster head election algorithm in LEACH is as follows. All the sensor nodes choosing a random number between $0 \sim 1$, and if it is less than a threshold $\mathrm{T}(\mathrm{n})$, the sensor nodes will broadcast an announcement message to notify others that it is a cluster head. In each round, if a node has been elected as a cluster head, its $T(n)$ is set to zero, so that the node will not be elected as a cluster head again. T(n) can be expressed as:

$$
T(n)=\left\{\begin{array}{l}
\frac{P}{1-P \times[r \bmod (1 / P)]}, n \in G \\
0, \quad \text { otherwise }
\end{array}\right.
$$

Where $\mathrm{P}$ is the desired percentage of cluster heads in the network (usually $\mathrm{P}$ is 0.05 in [5]), $\mathrm{r}$ is the current round, and $\mathrm{G}$ is the set of nodes that have not been cluster-heads in the last $1 / \mathrm{p}$ rounds. Using this threshold, each node will be a cluster-head at some point within $1 / p$ rounds. Nodes that have been cluster-head cannot become cluster-heads for a second time for $\mathrm{P}$ round. After that, each node has a $1 / \mathrm{p}$ probability of becoming a cluster head and joins that cluster to transmit data. The cluster heads combine and compress the data and forward it to the base station, therefore it extends the lifetime of major nodes.
In this algorithm, the energy consumption will allocate approximately uniformly among all nodes and the non-head nodes are turning off as much as possible. LEACH assumes that all nodes are in range of wireless transmission of the base station which is not the case in many sensor deployments. Time Division Multiple Access(TDMA) is deployed for better management and scheduling.

C. Radio energy dissipation model

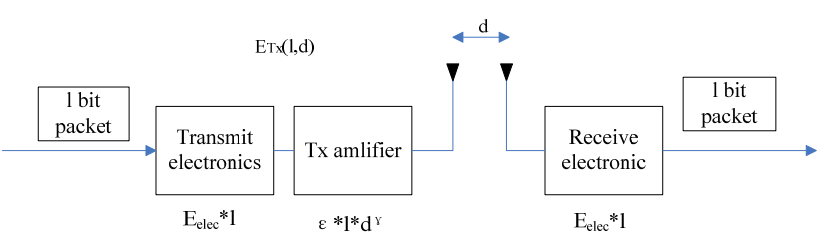

Figure 2. Radio energy dissipation model

According to the model shown in Fig.2. We assume that sensor nodes are randomly distributed in a square area of a WSN which has the following properties:

- Include two kinds of nodes: 1) Sensor nodes with limited energy can sense their own residual energy and have the same architecture; 2) One BS without energy restriction is far away from the area of sensor nodes.

- All sensor nodes are immobile. They use the direct transmission or multi-hop transmission to communicate with the BS.

- Sensor nodes sense environment at a fixed rate and always have data to send to the BS.

- The lifetime of WSN is the total amount of time before the first sensor node runs out of the power [6].

Equations (2) and (3) of energy consumption are adopted to calculate the transmission cost $\mathrm{E}_{\mathrm{Tx}}(\mathrm{l}, \mathrm{d})$ and the receiving cost $E_{R x}$ (1) of k-bit message between two nodes where their distance is $d$. Then the energy expenditure for transmitting L-bit message to distance is:

$$
E_{T x}(l, d)=l * E_{\text {elec }}+l * \varepsilon * d^{\gamma}
$$

And the energy expenditure for receiving L-bit message is:

$$
E_{R x}(l)=l * E_{\text {elec }}
$$

In Eq. (2) and Eq. (3), the electronics energy expenditure for one bit, and the value of $E_{\text {elec }}$ depends on factors such as the digital coding, modulation, filtering and spreading of the signal. Whereas the amplifier energy expenditure for one bit, the value of $\varepsilon^{*} \mathrm{~d}^{\gamma}$ depend on the distance from the sender to the receiver and the acceptable bit-error rate. In this paper, the channel model in the cluster is free space propagation model, and the channels between cluster head nodes and the sink node are multi-path fading channel. For the free space propagation , $\gamma$ is 2 , and $\varepsilon$ is denoted as $\varepsilon_{\mathrm{fs}}$. For multi-path fading channel, ris 4 , and eis denoted as $\varepsilon_{\mathrm{mp}}$. 


\section{ANALYZING THE PROBLEM OF LEACH AND WORK DONE}

\section{A. The problem of $\mathrm{LEACH}$}

LEACH is the simplest hierarchical protocol which possesses clustering approach and if implemented properly, can lead to energy efficient networking in WSN. And LEACH saves nodes' energy and prolongs network lifetime, but it still has some shortcomings as described below:

- $\quad$ LEACH is suitable for small size network because in LEACH it assumes that all nodes can communicate with each other and are able to reach sink which is not always true for large size network [7][9].

- At the beginning of each round, LEACH randomly selects the cluster heads without considering the residual energy of nodes. As a result, the number and location of cluster heads are unstable. With the increase of the time of round, the standard deviation of residual energy of cluster heads are also increased Then if the node with little residual energy is elected as a cluster head, it will run out of power quickly because of the heavy workload and then speed up the lifetime of network.

- Each cluster head using LEACH directly communicates with the BS no matter the distance is near or not. When the network is huge, the communication between cluster heads and BS consumes a lot of energy for the long distance transmission. So this nodes would dead quickly and the lifetime of WSN would be shorted. If something is wrong with the only channel, the data can not arrive at the $\mathrm{BS}$ in time.

\section{B. Improvement on LEACH}

\section{1) Cluster head election}

At the stage of setting up the cluster, in order to avoid the node with little residual energy is elected as a cluster head, take the residual energy into consideration when we calculate the value of $T(n)$.

After upper stage setting up the clusters, the cluster head will be rotated by computing the residual energy of nodes and select the node with maximal energy. This is helpful to uniformly distribute the load among the nodes in one cluster. The cluster heads can save energy since that they only broadcast information within their own clustering area whereas the cluster heads in LEACH broadcast the information for the whole networks.

\section{2) Data transmission phase}

During the data transmission phase, the common nodes in one cluster send data by the TDMA schedule to the cluster head which is similar in LEACH. And in LEACH each cluster head directly communicates with sink no matter the distance between cluster head and sink is far or not. But in this paper the transmission between cluster heads and the BS has two types: one-hop transmission like LEACH and multi-hop transmission.

This paper is focus on this, the objects is to make the loss of the transmission to the minimum. When the near cluster is alive and has enough residual energy, the far area's cluster heads may transmit their aggregated data to this near cluster head, and then this near cluster heads select more closer cluster head to receive the aggregated data, like this step by step transmit the data to BS. The algorithm in this paper selects optimal path and adopts multi-hop between cluster head and sink is presented here.

The cluster head which need to selects the next-hop node instead of transmitted the data to BS directly need meet the following conditions:

a) The distance to the BS is far, and between the BS there are another cluster heads with enough residual energy to transmit the aggregated data.

b) If this cluster heads node can transmit the data that has received from their own cluster, but this may lead to exhaust their own and caused nodes death.

\section{SIMULATION ANALYSIS}

We simulated our improvement protocol and compared the new one with the original LEACH protocol in this section.

\section{A. Simulation parameters seting}

1) In the network there have two kinds of nodes: sink nodes(BS, no energy restriction) and common nodes(another nodes, with restriction energy).

2) Suppose that every node knows its position,channels between sensor nodes are ideal, sending energy consumption is the same as receiving energy consumption,energy consumption in each round is $0.05 \mathrm{~J}$, the initial energy of the node in the network is 10J.

3) Nodes are randomly distributed in a area within 100 $\times 100 m$.

4) Probability of being cluster gead equals 0.04 in thie network.

5) Network with same number of nodes still may have different performance due to different network structure. In our paper simulation, the network topology is randomly built each time,and the result are the averaged for 5 different network topologies.

\section{B. Simulation results}

Fig.3 showed the performance of residual energy of LEACH protocol, and our new modified algorithm. From the result we know the new modified algorithm has more residual energy than LEACH. So our new algorithm has a better performance than LEACH protocol.

Fig.4 showed the performance of network death time for different number of nodes. The new modified algorithm survives longer than LEACH protocol and the result show that our new modified algorithm prolong the network lifetime as compared with the commonly used LEACH.

\section{CONCLUSION}

The modified LEACH protocol is presented in this paper, the new algorithm adopts multi-hop communication between cluster and BS. Simulation results show that this 
new algorithm has better performance than LEACH protocol.

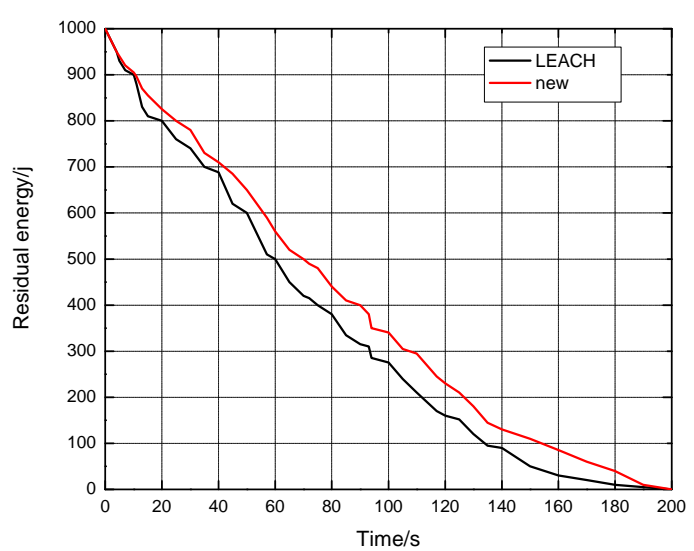

Figure 3. Residual energy with 100 nodes

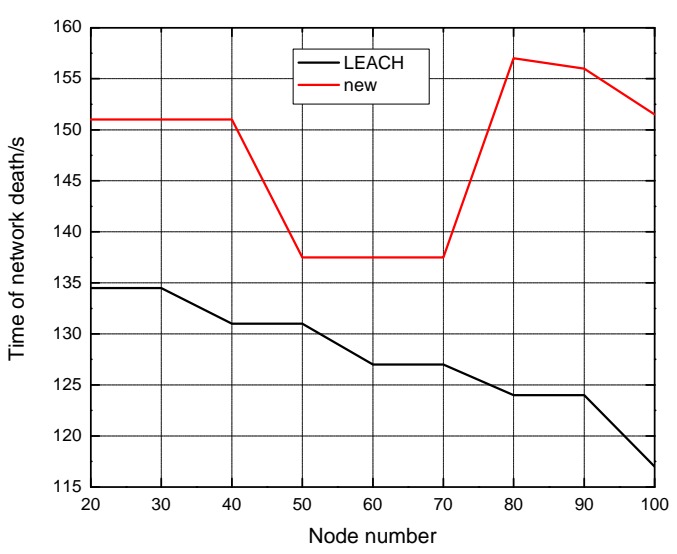

Figure 4. Time of network death in different number of node

\section{ACKNOWLEDGMENT}

We would like to take this opportunity to thank the anonymous reviewers for their valuable suggestion. This paper is supported by Institute of Telecom Services Support Systems Research, CQUPT.

\section{REFERENCES}

[1] W. R. Heinzelman, A. Chandrakasan and H. Balakrishnan, "Energy Efficient Communication Protocol for Wireless Micro sensor Networks", Proceedings of the $33^{\text {rd }}$ Hawaii International Conference on System Sccience,2000,1-10.

[2] J. N Al-Karaki, and A. E. Kamal, "Routing techniques in wireless sensor networks: a survey", IEEE Wireless Communications, Vol. 11, No. 6,pp.6-28, December 2004.

[3] N. M. A. Latiff, C. C. Tsimenidis, and B. S. Sharif, "Performance Comparison of Optimization Algoriithm for Clustering in Wireless Sensor Network," IEEE International Conference on Mobile Adhoc and Sensor Systems, 2007, 1-4.

[4] W. R. Heinzelman, A. Chandrakasan and H. Balakrishnan, "An Applocation-Specific Protocol Architecture for Wireless Microsensor Networks," IEEE Transactions on Wireless Communications,2002, 1(4):662-666.

[5] H. Yang and B. Sikdar, "Optimal Cluster Head Selection in the LEACH Architecture", IEEE International Conference on Performance, Computing, and Communications, 2007, 93-100.

[6] X. Zhou, B. Qin and F. Xu, "Wireless sensor neteorks and security", National defense industry Press, 2007.

[7] Mahmood Ali and Sai Kumar Ravula, "Real-time Support and Energy Efficiency in Wireless Sensor Network," School of Information Science, Computer and Electrical Engineering Halmstad University.

[8] Shio Kumar Singh, M P Singh, D K Singh, "A Survay of Energyefficient Hierarchical Cluster-Based Routing in Wireless Sensor Networks," Int. J. of Advanced Networking and Applications, Vol: 02, Pages: 570-580, 2010.

[9] Praveen Kaushik, Jyoti Singhai, "Energy Efficient Routing Algorithm for Maximizing the Minimum Lifetime of Wireless Sensor Network: A Review, " International Journal of Ad hoc, Sensor \& Ubiquitous Computing (IJASUC) Vol. 2, No. 2, June 2011

[10] Nidhi Barta, Anuj Jain, Surender Dhiman, "An Optimized Energy Efficient Routing Algorithm For Wireless Sensor Network," International Journal of Innovative Technology and Creative Engineering(ISSS:2045-8711) Vol. 1, No. 5,May 2011 